\title{
Últimas tendencias en la investigación sobre estructura de capital (periodo 2009-2018)
}

Latest trends in research on capital
structure (period 2009-2018)

Carmen Verónica Barón-Tinjacá*

* Magíster en Finanzas. Profesional en operaciones financieras Ecopetrol S.A. Bogotá (Colombia). [veronicabaron@gmail.com], [ORCID: 0000-0002-8455-3395].

Artículo recibido el 12 de noviembre de 2020.

Aceptado el 08 de diciembre de 2020.

Para citar este artículo:

Barón-Tinjacá, C. V. (2020). Últimas tendencias en la investigación sobre la estructura de capital (periodo 2009-2018). ODEON, 19, 7-30.

DOI: https://doi.org/10.18601/17941113.n19.02 


\section{Resumen}

El desarrollo de un área de conocimiento a través de la investigación requiere establecer cómo ha sido su evolución, identificar hacia dónde se han orientado los esfuerzos investigativos y cotejar los diferentes enfoques que se han dado a un determinado problema.

Dentro del campo de las finanzas corporativas, la estructura de capital ha sido un tema desarrollado con múltiples enfoques, sin que aún tengamos sobre ella una teoría de general aceptación. Teniendo en cuenta estas aseveraciones, se considera importante conocer cuáles son las últimas tendencias en la investigación sobre la estructura de capital.

Este artículo presenta los resultados del análisis bibliométrico de las últimas tendencias en la investigación sobre estructura de capital, decenio comprendido entre los años 2009 al 2018, lo que permite hacer un diagnóstico de su avance y sirve de fuente de referencia para futuras investigaciones. Además, incluye una comparación de este periodo con los nueve años anteriores, entre los años 2000 y 2008, destacando las diferencias en el análisis cuantitativo de las poblaciones de cada uno de estos periodos.

Para el desarrollo investigativo, cuyo resultado es el presente artículo, se utilizó la bibliometría como metodología de investigación.

El proceso investigativo partió de la revisión histórica de las teorías sobre estructura de capital, incluida en el marco teórico, continuó con el proceso bibliométrico y terminó con el análisis y la comparación de resultados.

Como resultado de este proceso se encontró que las últimas tendencias en la investigación sobre estructura de capital se encuentran compuestas en gran parte por el análisis y la verificación de los determinantes de la estructura de capital, y por la confirmación o refutación de teorías anteriores en diferentes tipos de empresas o sectores económicos.

Palabras clave: estructura de capital; apalancamiento; deuda: bibliometría.

Clasificación JEL: G32, G11, M21.

\section{Abstract}

The development of an area of knowledge through research requires establishing how its evolution has been, identifying where research efforts have been directed and comparing the different approaches that have been given to a certain problem.

Within the field of Corporate Finance, capital structure has been a subject developed with multiple approaches, without yet having a generally accepted 
theory about it. Taking into account these assertions, it is considered important to know what the latest trends in capital structure research are.

This article presents the results of the bibliometric analysis of the latest trends in research on capital structure, the decade between 2009 and 2018, allowing a diagnosis of its progress to be made and serving as a reference source for future research. It also includes a comparison of this period with the previous 9 years, between 2000 and 2008, highlighting the differences in the quantitative analysis of the populations of each of these periods.

For the research development, the result of which is the present article, bibliometrics was used as a research methodology.

The investigative process started from the historical review of the theories on capital structure, included in the theoretical framework, continued with the bibliometric process and ended with the analysis and comparison of results.

As a result of this process, it was found that the latest trends in capital structure research are largely made up of the analysis and verification of the determinants of the capital structure and the confirmation or refutation of previous theories in different types of capital. companies or economic sectors.

Key words: Capital structure; leverage; debt; bibliometrics.

JEL classification: G32, G11, M21.

\section{Introducción}

Las teorías sobre estructura de capital tratan de explicar la forma en que las empresas eligen financiar sus inversiones, los elementos críticos que tienen en cuenta, el impacto que este tipo de decisiones tiene sobre sus partes interesadas, entre otras características que han hecho que este tema sea abordado desde diferentes perspectivas y que, a la fecha, no contemos con una teoría de general aceptación que explique los elementos que configuran la decisión de adoptar una estructura de capital para las empresas.

El tema analizado tiene relevancia dado que la estructura de capital es uno de los conceptos más debatidos en finanzas corporativas por su importancia en la gestión de las empresas, el cumplimiento de sus objetivos, su crecimiento y estabilidad. Una decisión equivocada sobre la determinación de esta estructura puede derivar en problemas para la supervivencia de la empresa, incluida la quiebra (Warner, 1977). 
A lo largo de la historia, el desarrollo de la estructura de capital, desde el periodo antiguo de las finanzas (antes de 1950), con Benjamin Graham y David L. Dodd en 1934, cuestionándose si el valor de una empresa puede ser alterado a través de variaciones arbitrarias en la estructura de capital (Rubinstein, 2006), y posteriormente Modigliani y Miller (1958) con su propuesta I, ha pasado por múltiples teorías con diferentes enfoques. Por ejemplo, las teorías que analizan mercados ineficientes, como la Teoría de las Señales (Leland y Pyle, 1977) y la Teoría de la Jerarquización (Pecking Order Theory) (Myers y Majluf, 1984), las teorías con enfoque comportamental, como la Teoría del Sesgo Emocional del $\mathrm{CEO}^{1}$ (Azouzi y Jarboui, 2012) y la Teoría del Comportamiento (Graham et al., 2013) entre otros enfoques. En consecuencia, y ante el abanico de orientaciones, el presente artículo pretende mostrar hacia dónde ha ido la investigación en los últimos años, si se ha enfocado en verificar o refutar teorías anteriores, si se han presentado nuevas teorías o modelos que encaucen el conocimiento hacia nuevos puntos de vista y en qué conceptos de las teorías se han desarrollado más investigaciones.

El presente artículo consta de marco teórico, en el cual se describen las principales teorías que se han generado a través de la historia de las finanzas; luego la explicación de la metodología de investigación utilizada, siguiendo con los resultados del análisis bibliométrico, comparación de resultados entre los periodos 2000-2008 y 2009-2018, análisis de la muestra y clasificación de categorías para el periodo 2009-2018, definición de las últimas tendencias en la investigación sobre estructura de capital y conclusiones.

\section{Marco teórico}

El concepto de estructura de capital se refiere a la forma como las inversiones de la empresa se encuentran financiadas, entre recursos propios y deuda, incluida la posibilidad de no tener deuda (Strebulaev y Yang, 2013).

Cuando se hace referencia a estructura de capital, no en todos los casos se habla de una estructura óptima dado que, así como no existe una teoría de general aceptación que explique los elementos que configuran la decisión de adoptar una estructura de capital para las empresas, no se cuenta con una definición de general aceptación sobre lo que se consideraría una estructura óptima.

1 Chief Executive Officer.

ODEON, ISSN: 1794-1113, E-ISSN: 2346-2140, N 19, julio-diciembre de 2020, pp. 7-30 
No obstante lo anterior, se encuentra la definición de Ross et al. (2014), quienes afirman que la estructura óptima de capital es la combinación ideal de deuda y capital accionario con la que las empresas maximizan su valor y minimizan el costo total de capital.

Han pasado más de 80 años desde que Benjamin Graham y David L. Dodd abordaron el tema de la estructura de capital en su libro Security Analysis: Principles and Technique, cuestionándose si el valor de una empresa puede ser alterado a través de variaciones arbitrarias en la estructura de capital, y más de 60 años desde que fueron publicados los primeros planteamientos teóricos por Modigliani y Miller sobre el mismo tema, conocidos como la propuesta I, considerada la teoría base del pensamiento moderno acerca de la estructura de capital. Esta afirma que, en ausencia de impuestos, costos de quiebra y asimetrías de la información de los agentes, lo que se conoce como mercado eficiente, el valor de una empresa no se ve afectado por la forma en que está estructurado su capital, relación entre recursos propios y endeudamiento, y que, por ende, no existe una estructura óptima de capital (Modigliani y Miller, 1958). Posteriormente, al introducir los impuestos dentro del modelo, sus mismos autores contradijeron sus conclusiones iniciales (Modigliani y Miller, 1963), atendiendo a la ventaja de endeudarse al considerar fiscalmente deducibles los intereses pagados por la deuda. Esta teoría sugiere que la estructura óptima de capital es aquella combinación de recursos propios y deuda que maximiza el valor de mercado de la empresa, minimizando el costo medio de los recursos. Las anteriores consideraciones fueron la base de la Trade-Off Theory, también conocida como Teoría del Óptimo Financiero o Teoría del Equilibrio, la cual afirma que el nivel de endeudamiento de las empresas depende de la valuación que se haga del beneficio fiscal de la deuda frente a sus costos (p. e. costos de transacción, de insolvencia, de monitoreo). Uno de los principales trabajos de este enfoque teórico lo realizó Myers (1977).

En 1976, Jensen y Meckling plantearon la Teoría de la Agencia, la cual trata la relación que se establece cuando el principal (dueño) delega ciertos derechos en el agente (administrador), para que este último actúe en nombre del primero, lo que implica un grado de delegación de autoridad del principal al agente, donde cada sujeto tiene sus propios intereses y ambos intentan maximizar su utilidad, por lo cual no siempre el agente actuará en beneficio del principal. Para contrarrestar esta situación se requiere establecer mecanismos de control e incentivos, lo que da origen a los costos de agencia: costos de control, costos de fidelidad 
y pérdida residual ${ }^{2}$. Esta teoría, con enfoque comportamental, se incluye como Teoría de la Estructura de Capital, dado que la estructura de propiedad y la relación de agencia que tiene una empresa pueden definir la estructura de capital utilizada (Jensen y Meckling, 1976; Fama y Jensen, 1983a, 1983b).

Siguiendo con hipótesis que analizan mercados ineficientes, encontramos la Teoría de la Jerarquización (Pecking Order Theory) propuesta por Myers y Majluf (1984) y la Teoría de las Señales sugerida por Ross (1977) y Leland y Pyle (1977). La primera afirma que en presencia de asimetrías de la información (diferencias en la disponibilidad de la información de operaciones o perspectivas de las compañías entre los participantes del mercado), las empresas eligen su financiamiento mitigando las brechas de información que existen entre los gestores de la empresa y los inversores. Así, se genera un orden jerárquico de preferencias que inicia por financiamiento interno (beneficios retenidos), luego financiamiento externo de corto y largo plazo, obligaciones convertibles y, por último, la emisión de acciones. Se considera que iniciar con financiamiento interno es lo más adecuado dado que es lo menos riesgoso. Luego, cuando se elige la deuda externa, se busca aquella que implique un menor riesgo para la empresa, principalmente en la calidad de la deuda y mejores costos de financiamiento (Myers y Majluf, 1984).

La Teoría de las Señales (Leland y Pyle, 1977), también basada en la asimetría de la información, considera que la emisión de deuda da una señal al mercado acerca del bienestar de la empresa y la bondad de sus inversiones.

Iniciando este milenio, encontramos la Market Timing Theory (Baker y Wurgler, 2002), esta teoría afirma que a las empresas generalmente no les importa si se financian con deuda o capital, simplemente eligen la forma de financiamiento que, en el momento de tomar la decisión, parece ser la más valorada por los mercados financieros.

Entre las últimas teorías reconocidas sobre estructura de capital, se ha dado importancia al enfoque conductual, en el cual se analizan las finanzas desde un punto de vista psicológico sobre la manera como se comportan las personas

2 Costos de control: costos en los que incurre el principal, con el fin de disminuir las diferencias entre lo realizado y lo esperado de parte del agente.

Costos de fidelidad: costos en que incurre el agente para garantizar al principal que su labor no se desviará de lo esperado.

Pérdida residual: pérdida que genera el agente al tomar decisiones que el principal en su lugar no tomaría. 
y la forma en que toman las decisiones, asumiendo que los seres humanos somos parcialmente racionales. Dentro de este enfoque encontramos la Teoría del Sesgo Emocional del CEO (Azouzi y Jarboui, 2012), la cual afirma que las decisiones de inversión tienen relación directa con aspectos psicológicos del director ejecutivo. Los sesgos emocionales están relacionados con razonamientos basados en sentimientos o emociones. Otra teoría dentro de las llamadas finanzas conductuales es la Teoría del Comportamiento (Graham et al., 2013), que considera al actor económico, inversor individual, como un ser irracional e incongruente en la toma de decisiones que involucran dinero y riesgo. Se considera una evolución del pensamiento financiero y sirve para comprender la ineficiencia de los mercados y, también, para entender el proceso de toma de decisiones que enfrentan los involucrados en asuntos relacionados con el dinero, en nuestro caso, la estructura de capital por elegir y su efecto en los mercados.

\section{Metodología}

La bibliometría es entendida como el análisis de las publicaciones de artículos científicos, con el ánimo de identificar las tendencias en el desarrollo del conocimiento y la transformación de una disciplina (Bouyssou y Marchant, 2011). El análisis bibliométrico es una técnica de investigación aceptada en múltiples campos como los negocios, nuevas tecnologías, elección pública o la infometría (Wagner et al., 2011).

Dentro de la revisión realizada no se encontró un trabajo similar al presente, que a través de bibliometría buscara identificar las últimas tendencias en la investigación sobre estructura de capital.

El proceso bibliométrico que originó el presente artículo inició con la selección de la base de datos Scopus para la búsqueda de información objeto de la investigación. Esta fue seleccionada por tratarse de una base de datos sólida, accesible y reconocida dentro del ambiente científico y académico para el desarrollo de análisis bibliométricos. Entre los investigadores se considera que Scopus y Web of Science son las mayores bases de datos (Pérez-Escoda, 2017), sin embargo, Scopus fue escogida dado que, en muchos casos, contiene un mayor número de publicaciones y su información tiene una alta correlación con la de Web of Science (de Granda-Orive et al., 2013).

Posteriormente, se definieron como palabras clave de búsqueda: "Capital Structure", "Leverage" y "Debt", utilizando el operador booleano AND entre ellas, de forma tal que cada uno de los resultados obtenidos contuvieran las tres 
palabras, independientemente del orden y de su posición relativa. Estas palabras fueron elegidas dado que son las que con mayor frecuencia se observan en los escritos del tema en estudio.

Las palabras clave se incluyeron en inglés dado que a nivel mundial la gran mayoría de artículos que cuentan con respaldo académico, una metodología rigurosa y revisión de pares, están hechos o traducidos a ese idioma.

Respecto a los artículos, el tipo de documentos por analizar y las áreas de estudio de interés: Economics, Econometrics and Finance, Business y Management and Accounting, son las áreas afines a nuestra materia de investigación, dentro de las que contiene Scopus.

Para evaluar las últimas tendencias en un área de conocimiento, dentro del ambiente científico en general, se considera que un lapso de cinco años podría ser suficiente, por ello se realizó una primera revisión con los criterios explicados para el periodo comprendido entre los años 2014 y 2018, y se obtuvo una población de 296 artículos. Con el ánimo de obtener una mayor muestra para el análisis cualitativo y la definición de tendencias, se amplió el periodo de búsqueda a un decenio, para obtener así una población de 527 artículos, con la cual se realizó el análisis bibliométrico cuantitativo de la población y el análisis cualitativo de la muestra.

Dentro del periodo no se tuvo en cuenta el año 2019, dado que por lo general los artículos tardan aproximadamente un año en ser revisados, aprobados y publicados.

Así, la ecuación definitiva que determinó los artículos con los cuales se desarrolló la investigación fue: TITLE-ABS-KEY ("capital structure" AND "leverage" AND "Debt") AND DOCTYPE (ar) AND PUBYEAR > 2008 AND PUBYEAR < 2019 AND (LIMIT-TO (SUBJAREA, "ECON") OR LIMIT-TO (SUBJAREA , "BUSI"))

La información obtenida de la búsqueda en la base de datos fue seleccionada de acuerdo con el indicador bibliométrico de calidad (Durieux y Gevenois, 2010), ordenando los artículos de mayor a menor cantidad de citaciones y limitando la muestra al porcentaje definido (10\% para este caso), con lo que se obtuvieron 53 artículos para análisis cualitativo de muestra. Este indicador no exige un mínimo de citaciones para que una publicación sea o no tenida en cuenta para su análisis cualitativo.

Posteriormente, se realizó el análisis cualitativo de la muestra para determinar la pertinencia de los documentos teniendo en cuenta que los artículos hicieran referencia a investigaciones, análisis de casos, nuevas teorías y enfoques 
en estructura de capital. Finalmente, se realizó el análisis de resultados y se generaron las conclusiones.

Es importante tener en cuenta que los resultados obtenidos y detallados en el presente artículo pueden tener sesgo debido a la ecuación de búsqueda y la base de datos utilizada.

\section{Resultados del análisis bibliométrico}

El promedio anual de artículos publicados en el decenio de estudio correspondió a 53 por año. La producción tiene tendencia positiva y presentó el pico de mayor producción en el año 2016 (tabla 1).

Tabla 1: Número de artículos publicados por año

\begin{tabular}{|c|c|c|}
\hline \multicolumn{3}{|c|}{ Población 527 artículos } \\
\hline Año & No. de artículos publicados & Participación en el total de publicaciones (\%) \\
\hline 2009 & 33 & 6,30 \\
\hline 2010 & 40 & 7,60 \\
\hline 2011 & 60 & 11,40 \\
\hline 2012 & 51 & 9,70 \\
\hline 2013 & 47 & 8,90 \\
\hline 2014 & 55 & 10,40 \\
\hline 2015 & 63 & 12,00 \\
\hline 2016 & 67 & 12,70 \\
\hline 2017 & 52 & 9,90 \\
\hline 2018 & 59 & 11,20 \\
\hline
\end{tabular}

Fuente: cálculos propios con base en los resultados de la búsqueda a través de Scopus.

Respecto de las revistas o publicaciones en las cuales se han incluido estos artículos encontramos que los 527 han sido publicados en 160 revistas, lo que indica que el interés científico por la estructura de capital se encuentra disperso en diversas publicaciones, 147 de las cuales tienen solo entre 1 y 2 artículos, lo que corresponde al $26 \%$ de la información analizada. 
Al revisar los medios con mayor número de artículos publicados encontramos que los cinco primeros tienen el $20 \%$ de los artículos estudiados (figura 1).

Figura 1: Número de artículos por publicación



Fuente: resultados de la búsqueda a través de Scopus.

Las tres primeras revistas están ubicadas en el primer cuartil del ranking publicado por SJR-SCImago Journal y Country Rank para 2018 (Scimago Journal and Country Rank, s. f.), lo que indica que tienen un alto factor de impacto, el cual corresponde a la media del número de veces que se cita un artículo publicado en ellas. Además, el Journal of Financial Economics es la número dos de dicho ranking. Lo anterior nos indica la calidad de las publicaciones donde encontramos la mayoría de los artículos de la población y su impacto en la comunidad científica. El comportamiento anual para estas publicaciones se observa en la figura 2.

Como podemos observar, el comportamiento en la cantidad de artículos publicados por las revistas top, para el tema y durante el decenio estudiado, es muy irregular.

En la tabla 2 se presentan los artículos con mayor número de citas de acuerdo con el indicador bibliométrico de calidad seleccionado para el análisis realizado. 
Figura 2: Número de artículos por publicación por año

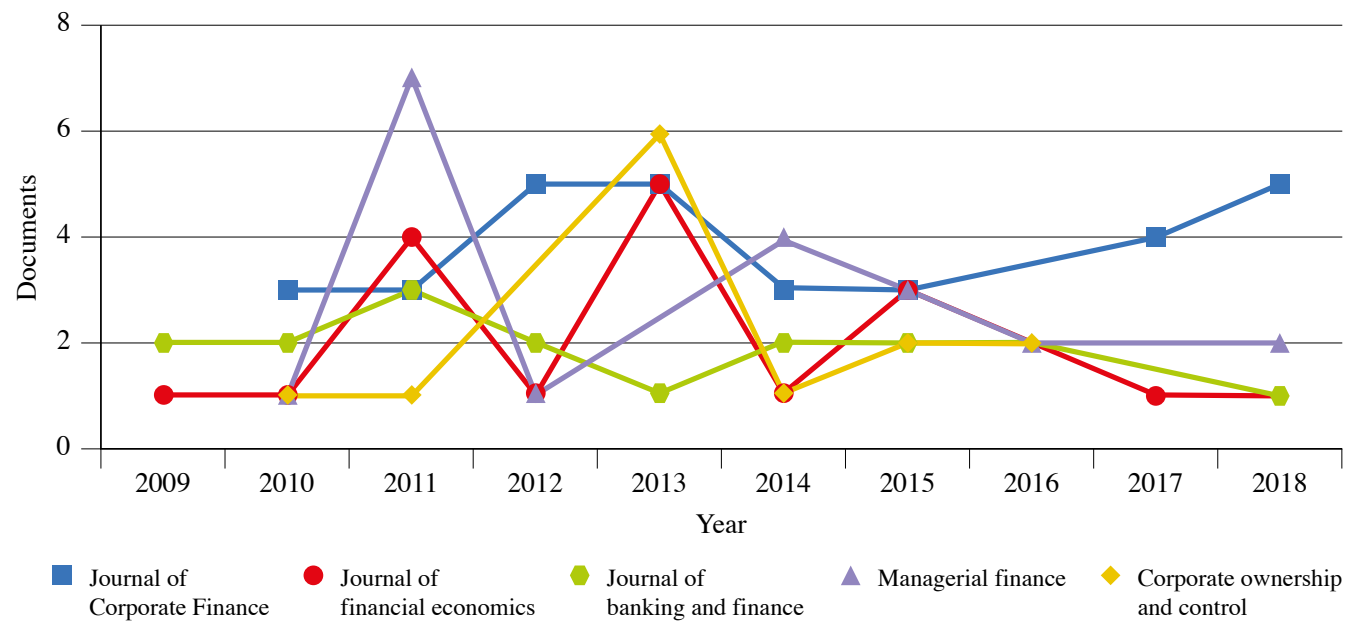

Fuente: resultados de la búsqueda a través de Scopus.

Tabla 2: Artículos más citados

\begin{tabular}{|l|l|c|}
\hline \multicolumn{1}{|c|}{ Autores } & \multicolumn{1}{|c|}{ Artículo } & No.de citas \\
\hline $\begin{array}{l}\text { Joseph P. H. Fan, Sheridan } \\
\text { Titman,Garry Twite }\end{array}$ & $\begin{array}{l}\text { An International Comparison of Capital Struc- } \\
\text { ture and Debt Maturity Choices }\end{array}$ & 247 \\
\hline Hui Chen & $\begin{array}{l}\text { Macroeconomic Conditions and the Puzzles of } \\
\text { Credit Spreads and Capital Structure }\end{array}$ & 166 \\
\hline Margaritis Dimitris,Maria Psillaki & $\begin{array}{l}\text { Capital Structure, Equity oOnership and Firm } \\
\text { Performance }\end{array}$ & 164 \\
\hline
\end{tabular}

Fuente: resultados de la búsqueda a través de Scopus.

En el artículo An International Comparison of Capital Structure and Debt

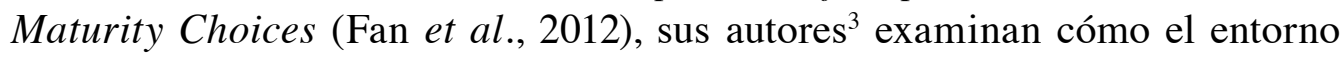
institucional influye en la estructura de capital y los plazos de vencimiento de la deuda analizando empresas de 39 países desarrollados y en desarrollo; los

3 Joseph P. H. Fan es profesor de la Escuela de Contabilidad y Departamento de Finanzas de la Universidad China de Hong Kong.

Sheridan Titman es profesor del Departamento de Finanzas en la Universidad de Texas. Garry Twite es profesor Honorario en el Departamento de Finanzas de la Universidad de Melbourne. 
autores concluyen que el sistema legal y tributario de un país, la corrupción y las preferencias de los proveedores de capital explican una porción significativa de la variación en los coeficientes de apalancamiento y vencimiento de la deuda.

En el artículo Macroeconomic Conditions and the Puzzles of Credit Spreads and Capital Structure, el profesor $\mathrm{Chen}^{4}$ (2010) propone un modelo dinámico de estructura de capital que demuestra cómo la variación del ciclo económico en las tasas de crecimiento esperadas, la incertidumbre económica y las primas de riesgo influyen en las políticas financieras de las empresas.

Los autores de Capital Structure, Equity Ownership and Firm Performan$c e^{5}$ (Margaritis y Psillaki, 2010) investigaron la relación entre la estructura de capital, la estructura de propiedad y el desempeño de la empresa utilizando una muestra de empresas manufactureras francesas, además probaron la relación directa entre apalancamiento y eficiencia estipulada por el modelo de costos de agencia de Jensen y Meckling (1976).

Al analizar el país de origen (entiéndase el país donde se desarrolló el artículo, por lo general el lugar de trabajo de los autores), podemos observar que el top 10 corresponde al $80 \%$ de los artículos analizados. Además, que Estados Unidos, país que lidera el escalafón con 159 artículos correspondientes al $30 \%$ del total, tiene el triple de producción que el segundo, Reino Unido con 53 artículos.

En total, los países europeos cuentan con 116 artículos dentro de la población analizada, en conjunto, una cifra menor a la de Estados Unidos.

Es notable la producción de los tres países asiáticos -Malasia, India y China-, que en total asciende a 100 artículos, interés que puede estar relacionado con su creciente desarrollo económico.

Al respecto de los países de Centro y Suramérica, el primero en aparecer en el ranking en el puesto 17 es Brasil con 10 publicaciones, Chile y Colombia con 2 artículos cada uno, en los puestos 43, 44 y Venezuela con 1 artículo en el puesto 69 (figura 3).

4 Hui Chen es profesor asociado de finanzas en la MIT Sloan School of Management.

5 Dimitris Margaritis es profesor del Departamento de Contabilidad y Finanzas de la Universidad de Auckland. Maria Psillaki es profesora del Departamento de Economía de Universidad del Pireo, Grecia. 
Figura 3: Número de artículos por principales países

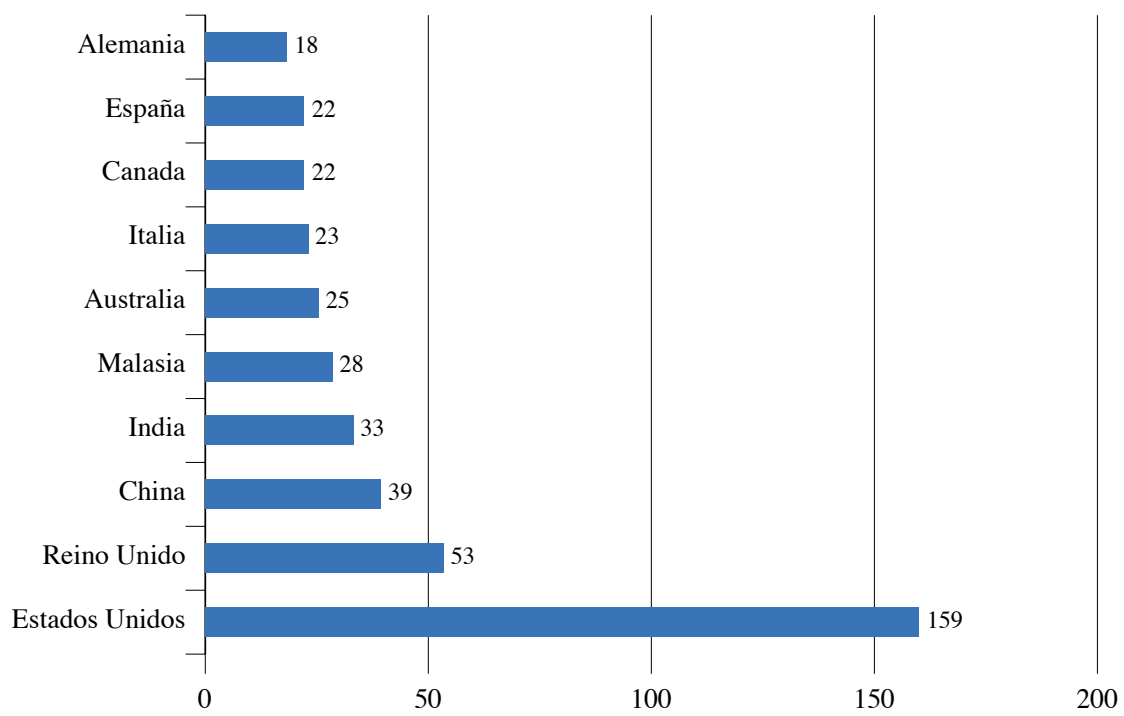

Fuente: resultados de la búsqueda a través de Scopus.

Pasando al análisis de afiliación de los artículos en estudio, encontramos que las universidades son las que generan la gran mayoría de conocimiento con el $98 \%$ de las publicaciones. El Fondo Monetario Internacional (FMI), el Rimini Center for Economic Analysis (RCEA), de Canadá, y el Banco de Canadá, son las únicas entidades diferentes a universidades presentes en nuestro análisis bibliométrico con ocho publicaciones.

En la figura 4 se presenta el listado de universidades con mayor número de publicaciones sobre estructura de capital entre los años 2009 y 2018.

Terminando la caracterización de la población de estudio, el análisis de los patrocinadores de artículos nos muestra que, de los 527 artículos, solamente el $17 \%$ aproximadamente fue patrocinado, lo que corresponde a 89 artículos. Los tres principales patrocinadores son asiáticos, aunque su peso en el total de artículos patrocinados es del $4 \%$; 46 instituciones apoyaron únicamente un artículo (figura 5). 
Figura 4: Número de artículos por principales filiaciones

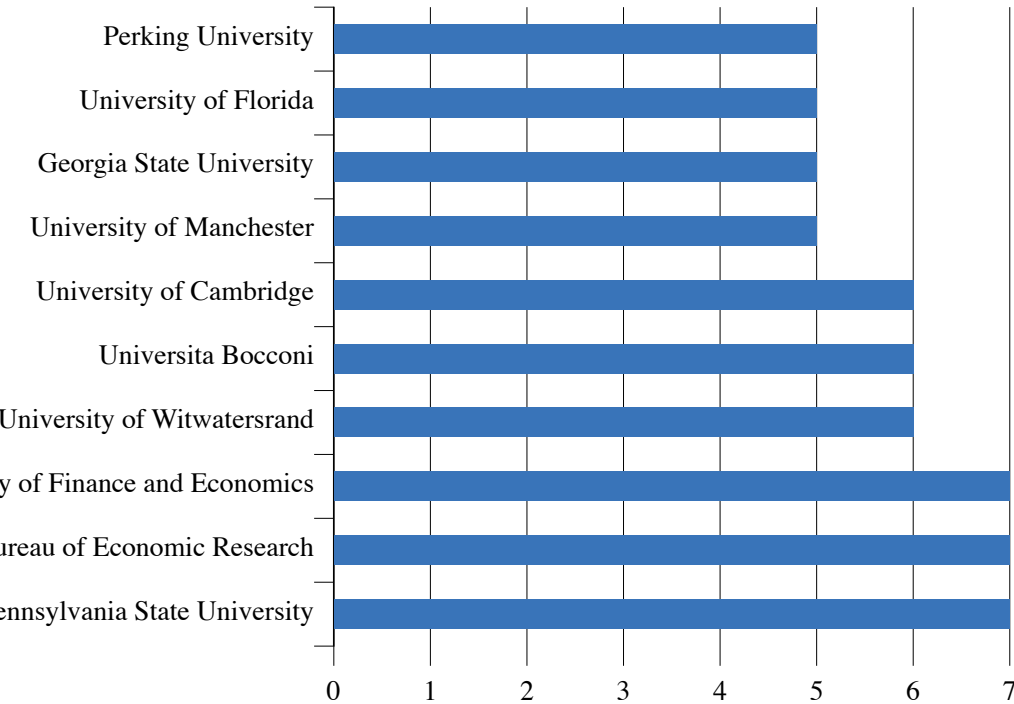

Fuente: resultados de la búsqueda a través de Scopus.

Figura 5: Número de artículos por principales patrocinadores

\section{Shanghai University}

of Finance and Economics

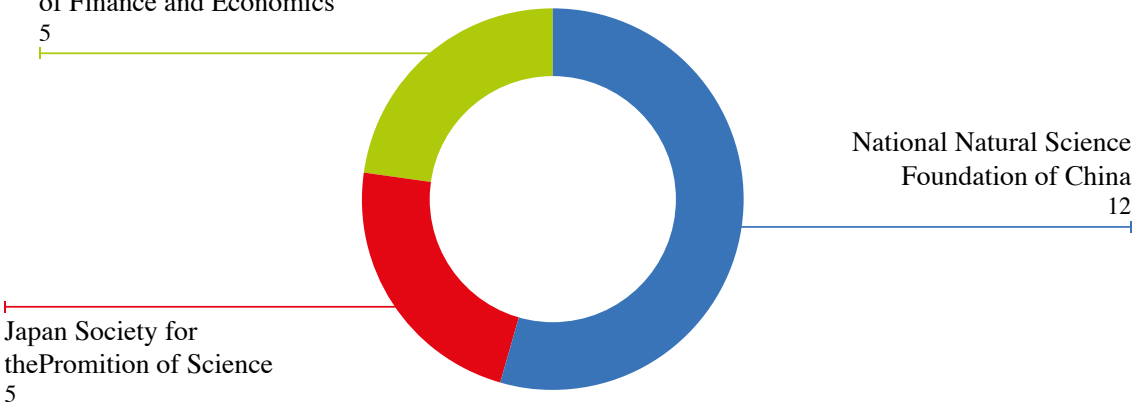

Fuente: resultados de la búsqueda a través de Scopus.

\section{Comparación del análisis bibliométrico: años 2000-2008 vs. 2009-2018}

Al comparar el último decenio con respecto a los nueve años anteriores, podemos observar que la publicación de artículos sobre estructura de capital ha presentado tendencia positiva. El número promedio de publicaciones entre los 
años 2000 y 2008 fue de 15 artículos por año, en contraste con el último decenio cuyo promedio anual aumentó más de 3 veces con 53 artículos (figura 6).

Figura 6: Número de artículos por año

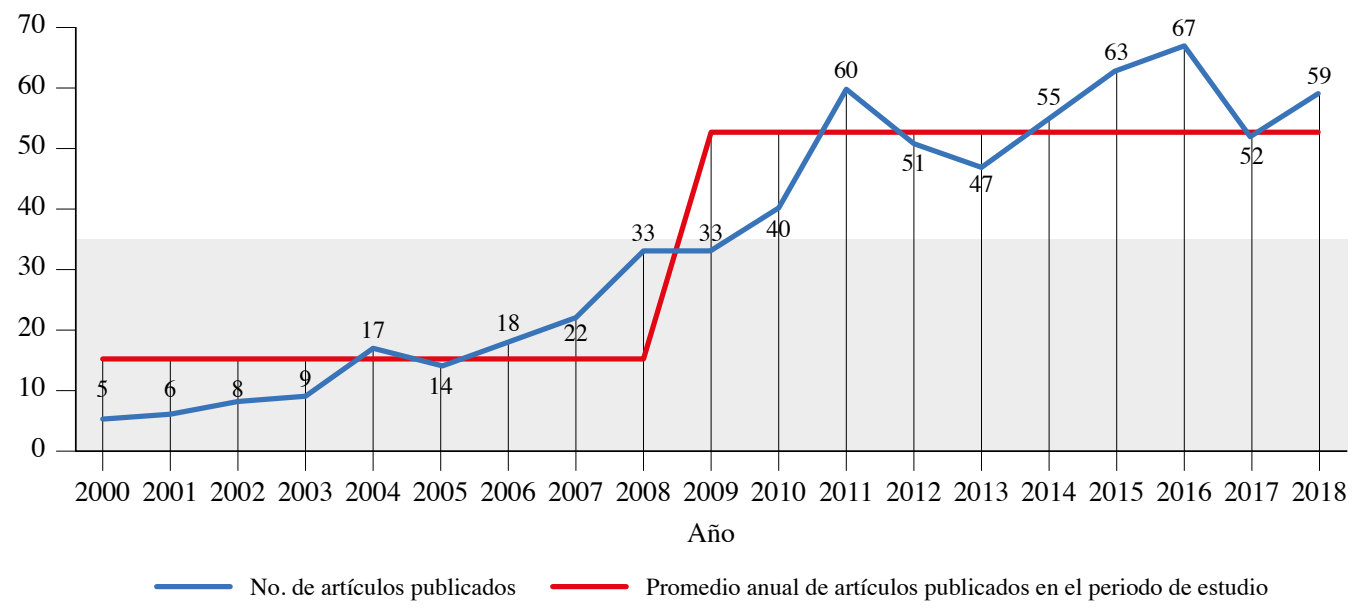

Fuente: resultados de la búsqueda a través de Scopus.

En cuanto a las revistas en las cuales se han publicado los artículos, en la figura 7 se puede observar que en el decenio analizado los artículos publicados aumentaron de manera significativa.

Figura 7: Comparación número de artículos por revista

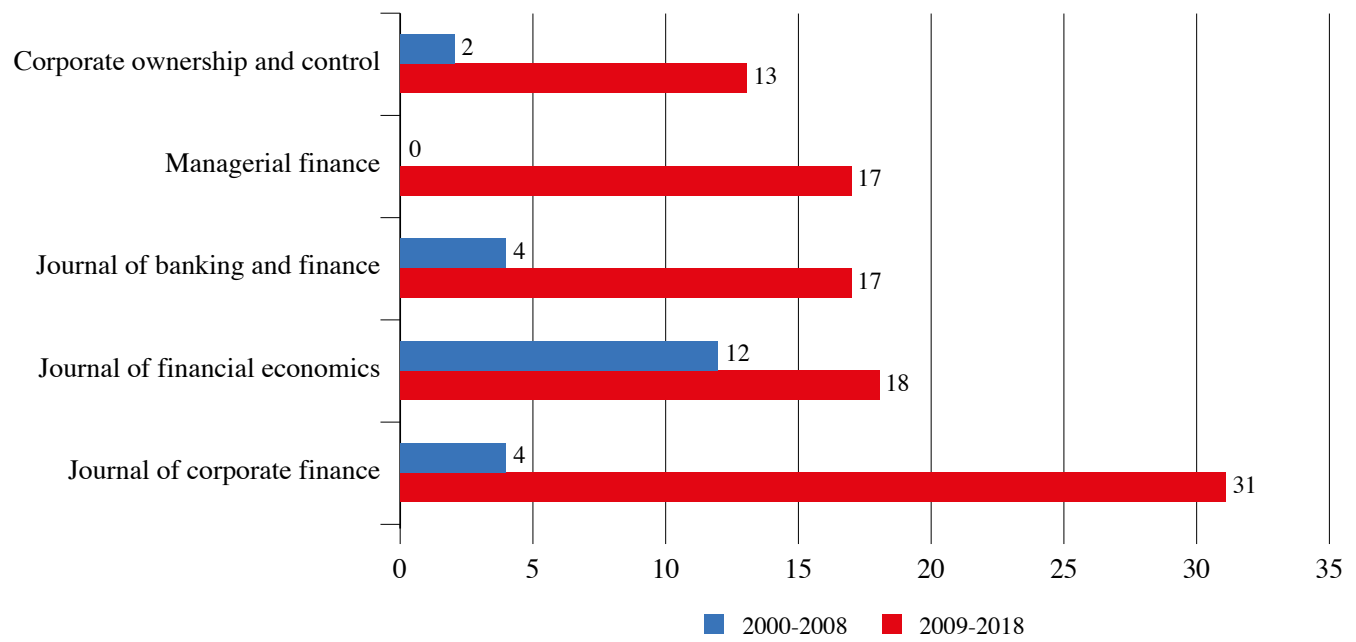

Fuente: resultados de la búsqueda a través de Scopus. 
Al revisar la producción de artículos por país, se observa que aquellos con mayor número en el decenio estudiado aumentaron, en promedio, más de 10 veces sus investigaciones (tabla 3 ).

Tabla 3: Comparación número de artículos por país

\begin{tabular}{|l|c|c|}
\hline \multicolumn{1}{|c|}{ País o territorio } & 2000-2008 & 2009-2018 \\
\hline Estados Unidos & 54 & 159 \\
\hline Reino Unido & 13 & 39 \\
\hline China & 3 & 33 \\
\hline India & 2 & 28 \\
\hline Malasia & 0 & 25 \\
\hline Australia & 2 & 23 \\
\hline Italia & 4 & 22 \\
\hline Canadá & 15 & 22 \\
\hline España & 5 & 18 \\
\hline Alemania & 2 & \\
\hline
\end{tabular}

Fuente: resultados de la búsqueda a través de Scopus.

Estados Unidos sigue a la vanguardia de la investigación. Malasia, después de no tener publicaciones, pasó a ser uno de los países top del ranking. Además, se observa que el interés científico en China, India, Australia y Alemania dio grandes pasos entre estos dos periodos.

En cuanto a la filiación de los artículos en estudio, encontramos que así como para el decenio 2009-2018 la gran mayoría de conocimiento, con el $98 \%$ de las publicaciones, fue generado por las universidades, entre los años 2000 y 2008 se presentaba también un nivel alto con el $97 \%$ de las publicaciones. Cabe anotar que para el periodo más reciente la National Bureau of Economic Research de Estados Unidos y el Centre for Economic Policy Research, de Reino Unido, no publicaron ningún artículo, mientras que en el periodo anterior habían publicado 7 y 5 respectivamente. 


\section{Análisis de la muestra}

Al realizar el análisis de los 53 artículos escogidos como muestra y seleccionados por el indicador bibliométrico de calidad, medido por el número de citaciones que han tenido los artículos (4.091) y correspondiente al $10 \%$ de la población objeto de estudio, se definieron cinco categorías para su clasificación, a saber:

1. Análisis de determinantes de la estructura de capital: corresponde a los artículos cuyo objetivo era plantear y demostrar la importancia de diferentes determinantes de capital.

2. Verificación/refutación de teorías sobre estructura de capital en país, región o sector industrial/comercial: artículos que exponen los resultados de la comprobación o refutación de teorías existentes respecto de estructura de capital, con información real de países, regiones o sectores industriales o comerciales.

3. Modelo propuesto: artículos que contienen modelos novedosos respecto a estructura de capital.

4. Capital objetivo: artículos que tratan acerca de la definición y explicación de capital objetivo y sus características.

5. Otros: artículos que por su contenido no se pueden clasificar en las cuatro categorías anteriores.

En la tabla 4 se presenta el resultado de la categorización.

Tabla 4: Categorización de la muestra

\begin{tabular}{|l|c|c|c|}
\hline \multicolumn{1}{|c|}{ Categorías } & $\begin{array}{c}\text { Artículos de } \\
\text { la muestra }\end{array}$ & $\begin{array}{c}\text { Citas de la } \\
\text { muestra }\end{array}$ & $\%$ \\
\hline Análisis de determinantes de la estructura de capital & 27 & 2029 & 49,60 \\
\hline $\begin{array}{l}\text { Verificación/refutación de teorías sobre estructura de capital } \\
\text { en país, región o sector industrial/comercial }\end{array}$ & 9 & 742 & 18,14 \\
\hline Modelo propuesto & 6 & 569 & 13,91 \\
\hline Capital objetivo & 7 & 481 & 11,76 \\
\hline Otros & 4 & 270 & 6,60 \\
\hline Total & 53 & 4091 & 100,00 \\
\hline
\end{tabular}

Fuente: elaboración propia. 


\section{6. Últimas tendencias en la investigación sobre estructura de capital}

De acuerdo con el análisis anterior podemos afirmar que gran parte de la investigación de los últimos años se ha orientado a explicar los diferentes determinantes de la estructura de capital. En la tabla 5 se presentan los diez determinantes con más citas realizadas, correspondientes al $77 \%$ del total de las citas en esta categoría.

Tabla 5: Clasificación de los determinantes de la estructura de capital

\begin{tabular}{|l|c|}
\hline \multicolumn{1}{|c|}{ Determinantes de la estructura de capital } & $\%$ \\
\hline $\begin{array}{l}\text { Entorno institucional del país o la región (sistema legal y tribu- } \\
\text { tario - factores macroeconómicos y corrupción) }\end{array}$ & 19,93 \\
\hline Estructura de propiedad & 11,22 \\
\hline Oportunidades de crecimiento & 10,02 \\
\hline Tipo de empresas & 8,03 \\
\hline Calificación crediticia & 6,39 \\
\hline Incentivos a la toma de riesgos gerenciales & 5,35 \\
\hline Tangibilidad & 5,07 \\
\hline Préstamos bancarios & 4,23 \\
\hline Compensación a los directivos & 3,71 \\
\hline Tamaño de la empresa & 3,43 \\
\hline
\end{tabular}

Fuente: elaboración propia.

Dentro de esta categoría se resaltan, además del artículo "An international comparison of capital structure and debt maturity choices" (Fan et al., 2012), del que tratamos en apartes anteriores, clasificado en el determinante de entorno institucional, los siguientes:

"National culture and capital structure decisions: Evidence from foreign joint ventures in China" (Li et al., 2011), este artículo es el resultado de la investigación por parte de los autores del papel del país de origen de las empresas en las decisiones de apalancamiento financiero. Analizaron un conjunto de datos de más de 8.000 empresas conjuntas extranjeras (con 32 países) en China, y presentan 
evidencia que respalda el importante papel de la cultura nacional en las decisiones de apalancamiento corporativo, prueba de la portabilidad de las culturas nacionales en un entorno donde las empresas conjuntas extranjeras operan y toman decisiones corporativas fuera de sus países de origen. Los resultados del documento respaldan la creciente conciencia entre los investigadores de finanzas de que las instituciones informales como la cultura, el nivel de confianza y el capital social son importantes en las decisiones financieras.

Respecto al determinante estructura de propiedad, el artículo "Ownership and capital structure in Latin America" (Céspedes et al., 2010) nos presenta los resultados del estudio realizado con información de 806 empresas latinoamericanas no financieras pertenecientes a siete países. Sus resultados apoyan la idea de que, a niveles altos de concentración de propiedad, la relación entre apalancamiento y propiedad es positiva, consistente con el argumento de que las empresas con propiedad altamente concentrada no buscan financiamiento de capital para evitar perder el control.

En la tabla 6 se presentan las teorías con mayor revisión (83\%) del total de citas en la categoría Verificación/refutación de teorías en país, región o sector industrial/comercial.

Tabla 6: Clasificación de las teorías verificadas/comprobadas

\begin{tabular}{|l|c|}
\hline \multicolumn{1}{|c|}{$\begin{array}{c}\text { Verificación / refutación de Teorías en país, } \\
\text { Región o sector industrial/comercial }\end{array}$} & $\%$ \\
\hline Pecking Order Theory & 33 \\
\hline Agency Theory & 25 \\
\hline Market Timing & 11 \\
\hline Trade Off Theory & 14 \\
\hline
\end{tabular}

Fuente: elaboración propia.

Se resalta el artículo "Further evidence on the capital structure of REITs" (Harrison et al., 2011) que contiene los resultados del estudio de las decisiones de estructura de capital de fideicomisos de inversión inmobiliaria (REIT) ${ }^{6}$, a través de una amplia muestra de observaciones de 2.409 empresas; el artículo

6 Real Estate Investment Trust, sociedades de inversión dueñas de activos inmobiliarios, cuyos ingresos proceden fundamentalmente de sus alquileres. 
concluye que la tangibilidad de los activos está positivamente relacionada con el apalancamiento y sugiere que la capacidad de endeudamiento de las empresas varía sistemáticamente con los mecanismos operativos y de financiamiento únicos empleados por los REIT, sus resultados proporcionan una mayor comprensión de las teorías de la estructura de capital de la competencia, en general respaldan las predicciones empíricas derivadas de las teorías de sincronización (Market Timing Theory) y compensación del mercado (Trade-Off Theory), aunque no respaldan las predicciones de la teoría del orden jerárquico (Pecking Order Theory).

El artículo "Capital structure, equity ownership and firm performance" (Margaritis y Psillaki, 2010), tratado en apartes anteriores, está considerado dentro de esta categoría verificación/refutación de teorías en país, región o sector industrial/comercial, Teoría de la Agencia, y cuenta con la mayor cantidad de citas (164) en esta categoría.

Dentro de la categoría: modelos propuestos, con el 13,91\% de la muestra, se destaca el artículo "Macroeconomic conditions and the puzzles of credit spreads and capital structure" (Chen, 2010), donde el autor propone un modelo dinámico de estructura de capital que demuestra cómo la variación del ciclo económico en las tasas de crecimiento esperadas, la incertidumbre económica y las primas de riesgo influyen en las políticas financieras de las empresas.

Dentro de la categoría Capital Objetivo, con el 11,76\% de la muestra, sobresale la publicación "Do firms have leverage targets? Evidence from acquisitions" (Harford et al., 2009), en la cual los autores, dentro del contexto de 1.188 grandes adquisiciones, evidencian que las empresas tienen estructuras de capital objetivo y los gerentes tratan de no alejarse demasiado de ellas, señalan cómo las desviaciones de ese objetivo afectan la forma en que eligen financiar una adquisición y cómo ajustan su estructura de capital después de la misma.

\section{Conclusiones}

Producto del proceso investigativo, cuyo objetivo principal era establecer las últimas tendencias en la investigación sobre la estructura de capital, se concluye que para el decenio comprendido entre los años 2009 al 2018, la investigación sobre estructura de capital se ha centrado en analizar y verificar sus elementos explicativos o determinantes en diferentes tipos de empresas, frente a lo cual han predominado los determinantes de carácter cualitativo como son la cultura, el nivel de corrupción, el entorno institucional, fiscal y legal de un país o región. 
Siguiendo el enfoque de finanzas conductuales, factores determinantes como el bienestar de los empleados y la compensación a los directivos también han sido analizados.

Otra gran parte de la investigación se ha orientado en confirmar o refutar teorías anteriores en diversos tipos de empresas o sectores económicos, la Teoría del Orden Jerárquico es la más abordada.

Los nuevos modelos propuestos recogen teorías anteriores enfocándolas en condiciones diferentes y, en general, intentando explicar el impacto de los determinantes o la conformación de una estructura óptima de capital. Se resalta la propuesta de modelo dinámico de estructura de capital.

A nivel internacional se evidenció el aumento en la investigación sobre la estructura de capital en los últimos años y la diversificación de temas al respecto. En cuanto a Centro y Suramérica, la producción científica sobre este tema es mínima y, contrario a la tendencia mundial, no ha aumentado.

Los resultados obtenidos sirven como fuente de referencia para futuras investigaciones, las cuales podrían, entre otras, orientarse hacia las variaciones planteadas por los nuevos modelos propuestos, la comparación de los resultados obtenidos con información de la base de datos de Scopus frente a resultados de otras bases de datos, el análisis de los determinantes de tipo conductual que han cobrado gran relevancia, el estudio del entorno institucional colombiano y su influencia en la estructura de capital de las empresas nacionales o extranjeras en el país, la aplicabilidad del modelo dinámico de estructura de capital (Chen, 2010) en las empresas nacionales, realizar un estudio seminal del desarrollo de las tendencias acá presentadas, entre otras.

\section{Referencias}

Azouzi, M. A. y Jarboui, A. (2012). CEO emotional bias and investment decision, bayesian network method. Business Excellence and Management, 2, 1259-1278.

Baker, M. y Wurgler, J. (2002). Market timing and capital structure. The Journal of Finance, 57, 1-32.

Bouyssou, D. y Marchant, T. (2011). Bibliometric rankings of journals based on impact factors: An axiomatic approach. Journal of Informetrics, 5, 75-86. 
Céspedes, J., González, M. y Molina, C. A. (2010). Ownership and capital structure in Latin America. Journal of Business Research, 63(3), 248-254.

Chen, H. (2010). Macroeconomic conditions and the puzzles of credit spreads and capital structure. Journal of Finance, 65, 2171-2212.

De Granda-Orive, J. I., Alonso-Arroyo, A., García-Río, F., Solano-Reina, S., JiménezRuiz, C. A. y Aleixandre-Benavent, R. (2013). Ciertas ventajas de scopus sobre web of science en un análisis bibliométrico sobre tabaquismo. http://redc.revistas. csic.es/index.php/redc/article/view/793/932.

Durieux, V. y Gevenois, P. A. (2010). Bibliometric indicators: Quality measurements of Scientific Publication. Radiology, 255, 342-351.

Fama, E. F. y Jensen, M. C. (1983a). Agency problems and residual claims. The Journal of Law and Economics, 26, 327-349.

Fama, E. F. y Jensen, M. C. (1983b). Separation of ownership and control. The Journal of Law and Economics, 26, 301-325.

Fan, J. P. H., Titman, S. y Twite, G. (2012). An international comparison of capital structure and debt maturity choices. Journal of Financial and Quantitative Analysis, 47, 23-56.

Graham, J. R., Harvey, C. R. y Puri, M. (2013). Managerial attitudes and corporate actions. Journal of Financial Economics, 109, 103-121.

Harford, J., Klasa, S. y Walcott, N. (2009). Do firms have leverage targets? Evidence from acquisitions. Journal of Financial Economics, 93, 1-14.

Harrison, D. M., Panasian, C. A. y Seiler, M. J. (2011). Further evidence on the capital structure of REITs. Real Estate Economics, 39, 133-166.

Jensen, M. C. y Meckling, W. H. (1976). Theory of the firm manajerial behaviour, ageny cost and ownership structure. Journal of Financial Economics, 3, 305-360.

Leland, H. E. y Pyle, D. H. (1977). Informational asymmetries, financial structure, and financial intermediation. The Journal of Finance, 32, 371-387. 
Li, K., Griffin, D., Yue, H. y Zhao, L. (2011). National culture and capital structure decisions: Evidence from foreign joint ventures in China. Journal of International Business Studies, 42, 477-503.

Margaritis, D., y Psillaki, M. (2010). Capital structure, equity ownership and firm performance. Journal of Banking and Finance, 34, 621-632.

Modigliani, F. y Miller, M. (1958). The cost of capital, corporation finance and the theory of investment. The American Economic Review, 48, 261-297.

Modigliani, F. y Miller, M. H. (1963). Corporate income Taxes and the cost of capital: A correction. The American Economic Review, 53, 433-443.

Myers, S. C. (1977). Determinants of corporate borrowing. Journal of Financial Economics, 5, 147-175.

Myers, S. C. y Majluf, N. S. (1984). Corporate financing and investment decisions when firms have information that investors do not have. Journal of Financial Economics, 13, 187-221.

Pérez-Escoda, A.(2017).Wos y Scopus: los grandes aliados de todo investigador. https:// www.revistacomunicar.com/wp/escuela-de-autores/wos-y-scopus-los-grandesaliados-de-todo-investigador/.

Ross, S. A. (1977). The determination of financial structure: The incentive-signalling approach. The Bell Journal of Economics, 8, 23-40.

Ross, S., Randolph W, W. y Bradford D, J. (2014). Fundamentos de Finanzas Corporativas. McGraw Hill Education.

Rubinstein, M. (2006). A History of the Theory of Investment. John Wiley \& Sons, Inc.

Scimago Journal and Country Rank (s. f.). Journal Rankings. https://www.scimagojr. com/journalrank.php?year=2018.

Strebulaev, I. A. y Yang, B. (2013). The mystery of zero-leverage firms. Journal of Financial Economics, 109, 1-23. 
Wagner, C. S., Roessner, J. D., Bobb, K., Thompson, J., Boyack, K. W., Keyton, J. y Börner, K. (2011). Approaches to understanding and measuring interdisciplinary scientific research (IDR): A review of the literature. Journal of Informetrics, 165 , 14-26.

Warner, J. B. (1977). Bankruptcy costs: Some evidence. The Journal of Finance, 32, 337. 